This is an electronic reprint of the original article. This reprint may differ from the original in pagination and typographic detail.

\author{
Author(s): Edgren, Johanna; Rantanen, Taina; Heinonen, Ari; Portegijs, Erja; Alén, Markku; \\ Kiviranta, Ilkka; Kallinen, Mauri; Sipilä, Sarianna
}

Title: $\quad$ Effects of progressive resistance training on physical disability among older community-dwelling people with history of hip fracture

Year: $\quad 2012$

Version:

Please cite the original version:

Edgren, J., Rantanen, T., Heinonen, A., Portegijs, E., Alén, M., Kiviranta, I., Kallinen, M., \& Sipilä, S. (2012). Effects of progressive resistance training on physical disability among older community-dwelling people with history of hip fracture. Aging Clinical and Experimental Research, 24(2), 171-175. https://doi.org/10.1007/bf03325162

All material supplied via JYX is protected by copyright and other intellectual property rights, and duplication or sale of all or part of any of the repository collections is not permitted, except that material may be duplicated by you for your research use or educational purposes in electronic or print form. You must obtain permission for any other use. Electronic or print copies may not be offered, whether for sale or otherwise to anyone who is not an authorised user. 


\title{
Effects of progressive resistance training on physical disability among older community-dwelling people with history of hip fracture*
}

\author{
Johanna Edgren1,2, Taina Rantanen1,2, Ari Heinonen², Erja Portegijss3, Markku Alén4, Ilkka \\ Kiviranta 5,6, Mauri Kallinen ${ }^{7}$ and Sarianna Sipilä1,2 \\ 1 Gerontology Research Center, and 2Department of Health Sciences, University of Jyväskylä, Jyväskylä, \\ Finland, 3Department of Geriatric Medicine and Department of Public Health, Academic Medical Center, \\ University of Amsterdam, Amsterdam, Netherlands, ${ }^{4}$ Department of Medical Rehabilitation, Oulu University \\ Hospital and Institute of Health Sciences, University of Oulu, Oulu, Finland, 5Department of Orthopedics \\ and Traumatology, University of Helsinki, Helsinki, Finland, ${ }^{6}$ Central Finland Health Care District, \\ Jyväskylä, Finland, 7Department of Physiatrics, Central Finland Central Hospital, Jyväskylä, Finland
}

ABSTRACT. Background and aims: Hip fracture is a common trauma in older people, and often leads to decreased muscle strength and increased physical disability. This randomized controlled trial examined whether three months of progressive resistance training (PRT) can reduce physical disability among older people with a history of hip fracture. Methods: $A$ population-based sample of 60-85-year-old community-dwelling persons, with hip fractures sustained on average three years earlier, were enrolled in the study. Of 78 people participating in laboratory assessments, those without contraindications for participation in resistance training were randomly assigned to a training group (TG, $n=22$ ) or a control group (CG, $n=21$ ). $T G$ took part in resistance training for three months twice a week. Training focused on lower limb muscles. Disability was assessed by a validated questionnaire containing six questions on activities of daily living ( $A D L)$ and nine on instrumental activities of daily living (IADL). A sum score was calculated separately for both items. High scores indicated more difficulties. Group differences were analysed with the Mann-Whitney and Chi-square tests. The effects of PRT on disability were tested with the McNemar test and by covariance analysis (ANCOVA). Results: TG and CG were comparable with respect to gender, age, chronic diseases, BMI, time since fracture, self-reported health, and level of physical activity at baseline. The ADL sum score in TG was 1.8 (2.0) at baseline and 1.1 (1.3) after follow-up; in CG values were 1.7 (1.8) and 1.5 (1.8) (ANCOVA $\mathrm{p}=0.034)$. IADL sum scores in TG were 3.9 (4.6) at baseline and 2.2 (3.8) after follow-up, and in CG 3.4 (3.6) and 2.4 (2.3) (ANCOVA p=0.529). Conclusions: Progressive resistance training reduced self-reported difficulties in $A D L$, even several years after fracture. More research is still needed on how to prevent physical disability among community-dwelling older people, especially after hip fracture.

(Aging Clin Exp Res 2012; 24: 171-175)

2012, Editrice Kurtis

\section{INTRODUCTION}

Hip fracture is a common and severe trauma in older people, and often leads to a decrease in muscle strength and increase in physical disability, together with other adverse health and economic consequences for patients, their families and society $(1,2)$. The incidence of hip fracture increases with age, and the total number of fractures in the future is expected to rise due to population aging (2). Effective exercise and physical rehabilitation strategies need to be developed to reduce physical disability after hip fracture. Adequate muscle strength is essential in performing activities which are important in daily life $(3,4)$. Progressive resistance training (PRT) can improve muscle strength and muscle mass even in older adults $(5,6)$. In clinical populations, insufficient evidence exists as to whether improved muscle strength translates into reduced physical

\footnotetext{
"Some of the results were presented at the Nordic Congress of Gerontology, 31.5.10, Reykjavik, Iceland.

Key words: Disability, hip fracture, older adults, resistance training.

Correspondence: Johanna Edgren, MSc, Researcher, Gerontology Research Center, Department of Health Sciences, P.O.Box 35 (Viv), 40014 University of Jyväskylä, Finland.

E-mail: johanna.edgren@jyu.fi
}

Received November 16, 2010; accepted in revised form March 22, 2011. 
disability $(7,8)$. This study examined whether three months of PRT can reduce physical disability among older people with history of hip fracture.

\section{METHODS}

We report analyses of secondary outcomes of a randomized controlled trial (RCT) studying PRT in older people with a history of hip fracture (ISRCTN34271567). The study design and training protocol are reported more in detail by Portegijs et al. (9) and are briefly summarized here.

\section{Participants}

All 452 surviving 60- to 85-year-old patients with hip fracture in the period 1998-2004 were identified from patient records of the Central Finland Central Hospital (7). Of these, 132 agreed to be interviewed by telephone. Those not living independently, or those who had neurological or progressive severe illnesses, moderate to severe memory problems (MMSE $<21$ ) or inability to walk outdoors without another person's assistance were excluded $(n=54)$. A further 35 people were excluded after a health examination. Those who did not have contraindications for PRT and consented to participate in a RCT were randomly assigned to a training $(T G, n=22)$ or control group (CG, $n=21$ ).

\section{Measurements}

Disability was assessed by a validated questionnaire (10) containing six questions with a four-graded response scale on activities of daily living (ADL) (Table 1) (11) and nine questions with a five-graded response scale on in- strumental activities of daily living (IADL) (Table 1) (12). The questions assessed perceived difficulties in ADL and IADL, for example: "How do you manage in eating?" In both $\mathrm{ADL}$ and IADL items, the first four response alternatives were the same: 1) I manage without difficulty, 2) with some difficulty, 3) with great difficulty, 4) I can't manage without another person's assistance. IADL items had the additional alternative 5) I can't manage even when assisted. These categorical variables were re-coded into dichotomous (Difficulty/No difficulty) for further analysis. A sum score was calculated separately for ADL and IADL items with the original variables. The theoretical range of the $\mathrm{ADL}$ sum score was 0-24 and that of the IADL 0-45. Higher scores indicated more difficulties and zero indicated no difficulties.

The level of physical activity was assessed with the Grimby scale, a semi-quantitative scoring system for estimating physical activity on six levels (13). Those who reported doing only light physical activity or mainly sitting were considered sedentary, those who reported being fairly physically active for at least three hours a week were considered physically active.

Pain in the fractured leg was assessed by asking: "Do you have detrimental pain in your fractured leg?" Those who answered "Yes" were considered as having detrimental pain. Cognitive state was assessed by the Mini Mental State Examination (MMSE), a brief 30-point questionnaire used to screen for cognitive impairment (14).

\section{Training intervention}

The TG participated in individually tailored PRT twice a week (1-1.5 h) for three months in a senior gym.

Table 1 - Proportion of self-reported difficulties in ADL/ADL in training and control groups.

\begin{tabular}{|c|c|c|c|c|}
\hline & \multicolumn{2}{|c|}{$\begin{array}{l}\text { Training group } \\
(\mathbf{n}=\mathbf{2 1 - 2 2})\end{array}$} & \multicolumn{2}{|c|}{$\begin{array}{l}\text { Control group } \\
(n=20-21)\end{array}$} \\
\hline & Baseline & Follow-up & Baseline & Follow-up \\
\hline \multicolumn{5}{|l|}{ Activities of daily living (ADL) } \\
\hline Eating & $1 / 22$ & $1 / 22$ & $2 / 21$ & $3 / 21$ \\
\hline Transferring from/to bed 1 & $6 / 22$ & $0 / 22$ & $5 / 21$ & $4 / 21$ \\
\hline Dressing & $5 / 22$ & $2 / 22$ & $4 / 21$ & $2 / 21$ \\
\hline Bathing & $4 / 22$ & $2 / 22$ & $1 / 21$ & $0 / 21$ \\
\hline Cutting toe nails & $14 / 21$ & $14 / 21$ & $11 / 21$ & $11 / 21$ \\
\hline Toileting & $1 / 22$ & $0 / 22$ & $1 / 21$ & $1 / 21$ \\
\hline \multicolumn{5}{|c|}{ Instrumental activities of daily living (IADL) } \\
\hline Preparing food & $5 / 22$ & $2 / 22$ & $3 / 21$ & $1 / 21$ \\
\hline Doing laundry & $5 / 22$ & $5 / 22$ & $2 / 21$ & $0 / 21$ \\
\hline Shopping & $8 / 22$ & $5 / 22$ & $9 / 21$ & $7 / 21$ \\
\hline Coping with light house work & $7 / 22$ & $5 / 22$ & $6 / 21$ & $2 / 21$ \\
\hline Coping with heavy house work ${ }^{2}$ & $13 / 22$ & $6 / 22$ & $14 / 21$ & $15 / 21$ \\
\hline Handling medication & $3 / 22$ & $0 / 22$ & $1 / 21$ & $1 / 21$ \\
\hline Using the telephone & $2 / 22$ & $1 / 22$ & $1 / 21$ & $1 / 21$ \\
\hline Using public transportation & $11 / 22$ & $6 / 22$ & $7 / 20$ & $7 / 20$ \\
\hline Handling finances & $1 / 22$ & $0 / 22$ & $2 / 21$ & $1 / 21$ \\
\hline
\end{tabular}


Training was carried out in groups of 4-8 persons, supervised by an experienced physiotherapist. It focused on the lower limb muscles, the aim being to reduce asymmetrical strength deficits and to increase the strength and power of lower limb muscles (9). 1-RM was estimated at the start of PRT and after weeks 6 to 8 . Training intensity was adjusted individually throughout the training period, based on the latest 1-RM estimation. The effects of intervention on muscle strength and power have been reported by Portegijs et al., who found that it increased muscle strength and power, especially on the weaker side (9).

\section{Statistics}

Group differences were analysed with the Mann-Whitney test for continuous variables and the Chi-square test for categorical variables. The effects of PRT on disability were tested with the McNemar test for categorical variables and by covariance analysis (ANCOVA), with baseline values as covariates for continuous variables. Participants with missing data were excluded from analysis. All analyses were performed with SPSS 17.0 software (SPSS Inc.).

\section{Ethics}

The Ethical Committee of the Central Finland Health Care District approved the study. All participants gave their written informed consent before undergoing laboratory examinations.

\section{RESULTS}

At baseline, TG and CG were comparable with respect to gender (total 14 men, 29 women), age (74.4 46.7 years), chronic diseases $(2.5 \pm 1.4), \mathrm{BMI}\left(26.5 \pm 3.7 \mathrm{~kg} / \mathrm{m}^{2}\right)$, time since fracture $(3.4 \pm 2.2$ years), self-reported health (69\% reported excellent health), level of physical activity (58\% physically active), pain (59\% reported detrimental pain in the fractured leg) and cognitive status (average MMSE score: 26; range 21-29).

Results for physical disability are listed in Table 1. The most obvious changes were observed in transferring from/to bed and coping with heavy housework. In the TG, six persons had difficulty in transferring from/to bed at baseline but none reported difficulty at follow-up. Similarly, in heavy housework, seven persons in the TG had less difficulty at follow-up compared with baseline. In the $\mathrm{CG}$, no such improvement was observed. The ADL and IADL sum scores, standard deviations and ANCOVA results are shown in Figure 1. In TG, the change in the ADL sum score was $9.0 \%$ and in the IADL score $13.2 \%$. The respective values in $C G$ were $2.6 \%$ and $8.1 \%$.

\section{DISCUSSION}

This study focused on community-dwelling older people who had sustained a hip fracture on average three years earlier. Even several years after a hip fracture, PRT reduced
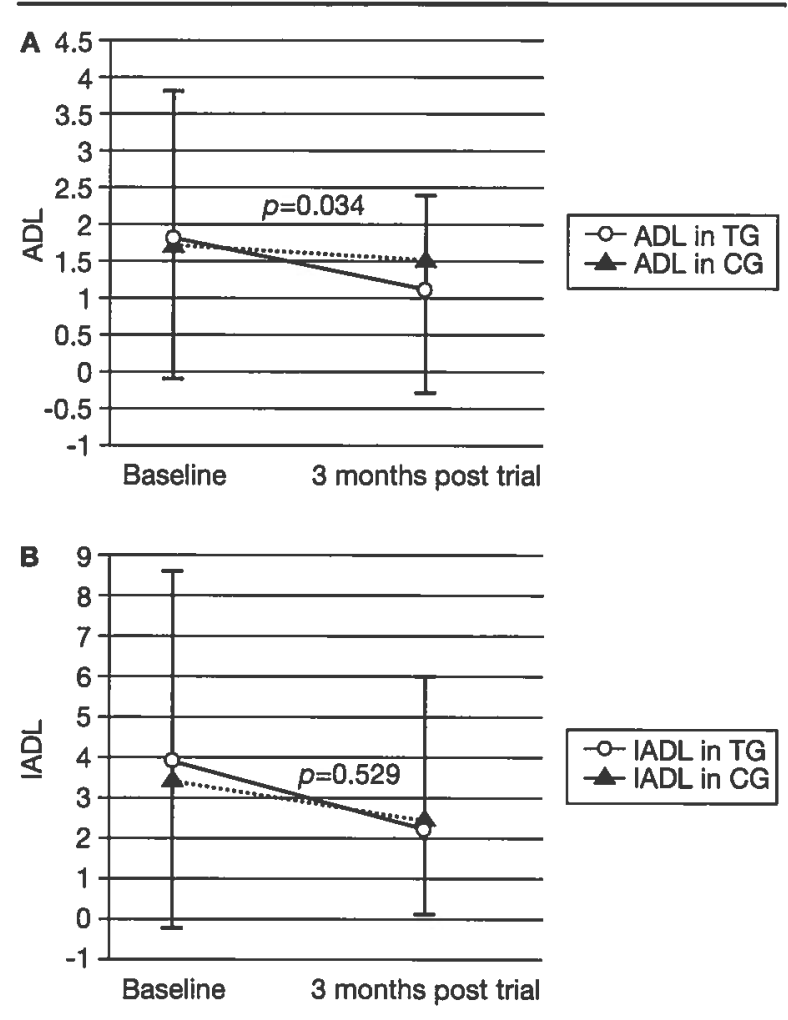

Fig. 1- $A D L(A)$ and IADL (B) sum scores, standard deviations and ANCOVA results in training (TG) and control (CG) groups adfusted for baseline values.

self-reported difficulties, especially in ADL. In particular, transferring to/from bed improved. Coping with heavy housework also substantially improved. These results were expected, since muscle strength of the lower limbs is required in these tasks. The effects of the intervention on muscle strength and power have previously been reported by Portegijs et al. (9). PRT improved both lower limb muscle force and power, particularly on the weaker side.

According to earlier studies, the results of self-reported measures of physical function are correlated with the results of performance-based measures in older people with hip fracture, and either type of functional measure would be suitable for use in clinical trials (15). For example, Kivinen et al. (16) found that ADL capacity and performance tests were significantly correlated among 70to 89-year-old men and that the risk of disability increased systematically with decreasing performance in every performance test. The authors stated that the choice of which measurement or assessment tool to use in assessing functional status should be based on the aim of the research and the study population (16). Self-reported disability reflects the results of activities undertaken over at least a couple of days, and may be based on a person's 
reliance on various aids or equipment. Instead, performance-based measurements examine performance only at a single point in time and do not reflect adaptations made in a person's daily living (17-19).

There are only a few studies on the effects of resistance training on physical disability among high-risk groups of older people. However, several studies have been published on the effects of resistance training on physical functional capacity assessed by performance-based measures. Self-reported difficulty (disability) and performance-based measurements (impairment or limitation) assess different stages of the disablement process (20-22). In this study, we concentrated especially on physical disability.

The recent systematic review by Liu and Latham (8) provides evidence that PRT is an effective intervention for improving physical functioning among older people, including strength and the performance of various simple and complex activities. This is in line with our results. More research is still needed on how PRT can be used with clinical populations, as adverse events have not been adequately reported (7). For example, in the study by Timonen et al. (23), PRT for ten weeks did not cause any serious adverse events, but neither had any positive effects on functional independence in frail older women, as measured by the ADL/ADL scale. In our study, no serious adverse events occurred, and the training group improved in functional independence.

To our knowledge, no similar studies have been carried out on a comparable population. Mangione et al. (24) reported the effects of PRT on physical function in older hip fracture patients, but used a different outcome variable, the physical function subscale of the SF-36 health survey. These authors showed that home-based moderate- to high-intensity PRT with a portable resistance exercise machine seemed to improve muscle force, endurance and gait, and also physical function, but the improvement was significant only for muscle force (24). Binder et al. (25) reported that, among older hip fracture patients, supervised physical therapy, including not only PRT but also flexibility, balance, coordination and movement speed exercises, enhanced physical performance and reduced physical disability, as measured on the ADL/AADL scale. Again, changes in ADL/IADL were nonsignificant (25). In our study, PRT of the lower limbs was effective, and improved independence in ADL/AADL among older people with hip fracture history.

To maximize the effect on interventions to reduce physical disability, factors which moderate or mediate the role of beliefs, emotions and coping strategies need to be examined (8). Keysor and Jette also emphasize the importance of contextual factors, including environmental and personal factors (26). However, scientific evidence of multi-component interventions targeted at physical disability is lacking, and more research is needed on how to prevent physical disability among community-dwelling older people, especially after hip fracture. It is also es- sential for responsive outcome measures to be used, whereas a larger sample size is preferable, to facilitate statistical analysis and determine whether exercise is actually effective in reducing physical disability.

\section{Strengths and limitations}

The present study has some limitations. The power calculations were initially carried out to detect changes in the strength variables. Thus, the sample size was slightly insufficient for the frequency data. However, some significant results were found. Especially considering the large heterogeneity of clinical populations such as ours, a larger sample size and longer duration of the PRT program may have shown clearer training effects (27). Another limitation is the lack of follow-up to detect long-term effects of the program and maintenance of results after three months of training.

The strengths of this RCT study are that it included a medical examination to ensure safe participation, and training was planned and carried out according to ACSM guidelines (28). This was also a community-based sample of frail older people who represented a specific clinical population. In addition, our primary outcome was perceived disability, a subjective evaluation of difficulties in daily living. Consequently these results should be considered clinically relevant and significant. The study of Portegijs et al. reported previously high feasibility and compliance in this RCT, and no serious adverse events occurred (9). Lastly, the training protocol of this study can be considered cost-effective, since training was group-based and thus profitable, compared with individual rehabilitation therapy by a physiotherapist.

\section{CONCLUSIONS}

Progressive resistance training reduced self-reported difficulties, especially in ADL, even several years after hip fracture. In particular, transferring to/from bed improved. Coping with heavy housework also substantially improved. More research is needed on how to prevent physical disability among community-dwelling older people, especially after hip fracture. To improve mobility and balance function, other problems such as fear of falling and issues like training specificity also need to be taken into account. It is also essential for responsive outcome measures to be used, and a larger sample size would be preferable, to facilitate statistical analysis and determine whether exercise is actually effective in reducing physical disability.

\section{ACKNOWLEDGEMENTS}

This study was supported by the Finnish Ministry of Education and Culture and the Juho Vaino Foundation.

Conflict of interest: None.

\section{REFERENCES}

1. Kannus P, Niemi S, Parkkari J, Palvanen M, Vuori I, Järvinen M. Nationwide decline in incidence of hip fracture. J Bone Miner Res 2006; 21: 1836-8. 
2. Lönroos $E$, Kautiainen $H$, Karppi $P$ et al. Increased incidence of hip fractures. A population based-study in Finland. Bone 2006; 39: 623-7.

3. Penninx BW, Messier SP, Rejeski WJ et al. Physical exercise and the prevention of disability in activities of daily living in older persons with osteoarthritis. Arch Intern Med 2001; 161: 2309-16.

4. Fielding RA, LeBrasseur NK, Cuoco A, Bean J, Mizer K, Fiatarone Singh MA. High-velocity resistance training increases skeletal muscle peak power in older women. J Am Geriatr Soc 2002; 50: 655-62.

5. Sipilä S, Multanen J, Kallinen M, Era P, Suominen H. Effects of strength and endurance training on isometric muscle strength and walking speed in elderly women. Acta Physiol Scand 1996; 156: 457-64.

6. Wieser $M$, Haber $P$. The effects of systematic resistance training in the elderly. Int J Sports Med 2007; 28: 59-65.

7. Liu CJ, Latham NK. Progressive resistance strength training for improving physical function in older adults. Cochrane Database Syst Rev 2009; (3)(3): CD002759.

8. Liu CJ, Latham N. Can progressive resistance strength training reduce physical disability in older adults? A meta-analysis study. Disabil Rehabil 2011; 33: 87-97.

9. Portegijs E, Kallinen M, Rantanen T et al. Effects of resistance training on lower-extremity impairments in older people with hip fracture. Arch Phys Med Rehabil 2008; 89: 1667-74.

10. Laukkanen P, Karppi P, Heikkinen E, Kauppinen M. Coping with activities of daily living in different care settings. Age Ageing 2001; 30: 489-94.

11. Katz S, Akpom CA. 12. index of ADL. Med Care 1976; 14 (5 Suppl): $116-8$.

12. Lawton MP, Brody EM. Assessment of older people: Self-maintaining and instrumental activities of daily living. Gerontologist 1969; 9: 179-86.

13. Grimby G. Physical activity and muscle training in the elderly. Acta Med Scand Suppl 1986; 711: 233-7.

14. Folstein MF, Folstein SE, McHugh PR. "Mini-mental state". A practical method for grading the cognitive state of patients for the clinician. J Psychiatr Res 1975; 12: 189-98.

15. Latham NK, Mehta V, Nguyen AM et al. Performance-based or self-report measures of physical function: Which should be used in clinical trials of hip fracture patients? Arch Phys Med Rehabil $2008 ; 89: 2146-55$.
16. Kivinen P, Sulkava R, Halonen P, Nissinen A. Self-reported and performance-based functional status and associated factors among elderly men: The Finnish cohorts of the seven countries study. J Clin Epidemiol 1998; 51: 1243-52

17. Guralnik JM, Branch LG, Cummings SR, Curb JD. Physical performance measures in aging research. J Gerontol 1989; 44: M141-6.

18. Myers AM, Holliday PJ, Harvey KA, Hutchinson KS. Functional performance measures: Are they superior to self-assessments? J Gerontol 1993; 48: M196-206.

19. Tinetti ME, Ginter SF. Identifying mobility dysfunctions in elderly patients. Standard neuromuscular examination or direct assessment? JAMA 1988; 259: 1190-3.

20. Nagi SZ. An epidemiology of disability among adults in the united states. Milbank Mem Fund Q Health Soc 1976; 54: 439-67.

21. Kelly-Hayes M, Jette AM, Wolf PA, D'Agostino RB, Odell PM. Functional limitations and disability among elders in the Framingham study. Am J Public Health 1992; 82: 841-5.

22. Guralnik JM, Simonsick EM, Ferrucci L et al. A short physical performance battery assessing lower extremity function: Association with self-reported disability and prediction of mortality and nursing home admission. J Gerontol 1994; 49: M85-94.

23. Timonen L, Rantanen T, Mäkinen E, Timonen TE, Törmäkangas $T$, Sulkava R. Effects of a group-based exercise program on functional abilities in frail older women after hospital discharge. Aging Clin Exp Res 2006; 18: 50-6.

24. Mangione KK, Craik RL, Tomlinson SS, Palombaro KM. Can elderly patients who have had a hip fracture perform moderateto high-intensity exercise at home? Phys Ther 2005; 85: 727 39.

25. Binder EF, Brown M, Sinacore DR, Steger-May K, Yarasheski KE, Schechtman KB. Effects of extended outpatient rehabilitation after hip fracture: A randomized controlled trial. JAMA 2004; 292: 837-46.

26. Keysor JJ, Jette AM. Have we oversold the benefit of late-life exercise? J Gerontol A Biol Sci Med Sci 2001; 56: M412-23.

27. Eastwood EA, Magaziner J, Wang J et al. Patients with hip fracture: Subgroups and their outcomes. J Am Geriatr Soc 2002; 50: 1240-9.

28. American College of Sports Medicine. ACSM's guidelines for exercise testing and prescription, 6th ed. Philadelphia: Lippincott Williams \& Wilkins; 2000. 